\title{
The EA-Dimension of a Commutative Ring
}

\author{
Mosbah Eljeri \\ Institut Préparatoire aux Études D’ingénieurs de Monastir, Tunisia \\ Correspondence should be addressed to Mosbah Eljeri; elljeri@yahoo.fr
}

Received 29 October 2013; Accepted 21 November 2013

Academic Editors: D. Anderson and A. V. Kelarev

Copyright (C) 2013 Mosbah Eljeri. This is an open access article distributed under the Creative Commons Attribution License, which permits unrestricted use, distribution, and reproduction in any medium, provided the original work is properly cited.

An elementary annihilator of a ring $A$ is an annihilator that has the form $(0: a)_{A} ; a \in R \backslash(0)$. We define the elementary annihilator dimension of the ring $A$, denoted by $\operatorname{EAdim}(A)$, to be the upper bound of the set of all integers $n$ such that there is a chain $(0$ : $\left.a_{0}\right) \subset \cdots \subset\left(0: a_{n}\right)$ of annihilators of $A$. We use this dimension to characterize some zero-divisors graphs.

\section{Introduction}

In this paper, all rings are considered to be commutative and unitary.

Let $A$ be a ring and $S$ be a nonempty subset of $A$. We call the annihilator of $S$ in $A$ denoted by $(0: S)_{A}$ or $(0: S)$ the set $\{a \in A / a S=(0)\}$. If $S=\{a\}$ is a singleton then $(0: S)$ will be denoted by $(0: a)$. If $a \neq 0$ then $(0: a)$ is called an elementary annihilator. An annihilator is said to be maximal if it is maximal in the set of all proper annihilators of $A$. It is well known that all maximal annihilators are elementary. For $n \in$ $\mathbb{N}$ an elementary annihilator chain $\left(0: a_{1}\right) \subset\left(0: a_{2}\right) \subset \cdots \subset$ $\left(0: a_{n+1}\right)$ is said to be a chain of elementary annihilators with length $n$ ending in $\left(0: a_{n+1}\right)$. The upper bound of the set of all lengths of elementary annihilator chains ending in $(0: a)$ is called the elementary annihilator height of $a_{n+1}\left(\right.$ or $\left.\left(0: a_{n+1}\right)\right)$. In this paper, we introduce a dimension of a ring $R$ using elementary annihilator chains called elementary annihilator dimension, denoted by $\operatorname{EAdim}(R)$. The $\operatorname{EAdim}(R)$ is the upper bound of the set of elementary annihilator heights. We use this dimension to study zero-divisor graphs.

We introduce a class of rings called isometric maximal elementary annihilator rings, in short IMEA-rings. That is the class of rings with finite EAdimension whose all maximal annihilators have the same height.

\section{Elementary Annihilator Dimension of a Ring}

Definition 1. (1) Let $n \in \mathbb{N}$ and $\left(0: a_{1}\right) \subset\left(0: a_{2}\right) \subset \cdots \subset$ $\left(0: a_{n+1}\right)$ be chain of elementary annihilators in the ring $A$.
One says that this chain is an elementary annihilator chain of length $n$ ending in $\left(0: a_{n+1}\right)$.

(2) Let $a$ be a nonzero element of $A$. One defines the elementary annihilator height of $a$, denoted by $\operatorname{EAht}(a)$, as the upper bound of the set of all lengths of elementary annihilator chains ending in $(0: a)$.

(3) One calls elementary annihilator dimension of $A$, denoted by $\operatorname{EAdim}(A)$, the upper bound of the set $\{\operatorname{EAht}(a) ; a \in A \backslash\{0\}\}$.

Example 2. (1) EAdim $(\mathbb{Z} / 4 \mathbb{Z})=1$. Indeed, $(0: 1) \subset(0: 2)$ is the longest chain of elementary annihilators in $\mathbb{Z} / 4 \mathbb{Z}$.

(2) $\operatorname{EAht}(1)=0$.

(3) All nonzero zero-divisors $a$ satisfy $\operatorname{EAht}(a) \geq 1$. Indeed, $(0: 1) \subset(0: a)$ is a chain of length one.

It is easy to check the following results.

Remark 3. (1) Let $a \in A \backslash\{0\}, \operatorname{EAht}(a)=0$ if and only if $a$ is regular.

(2) $\operatorname{EAdim}(A)=0$ if and only if $A$ is a domain.

(3) For an ideal $I$ of $A, \operatorname{EAdim}(A / I)=0$ if and only if $I$ is prime.

(4) If $a$ is a nonzero noninvertible element $\operatorname{EAdim}(A /(a))=0$ if and only if $a$ is prime.

We denote by nil $(A)$ the set of all nilpotent elements of $A$. $A$ is said to be reduced if it has no nilpotents other then zero.

Theorem 4. Let $a \in \operatorname{nil}(A) \backslash\{0\}$ and $n(a)$ be its index of nilpotency; one has: $\operatorname{EAht}(a)+n(a) \leq \operatorname{EAdim}(A)+2$. 
Proof. If $\operatorname{EAht}(a)+n(a)$ or $\operatorname{EAdim}(A)$ is infinite the result is obvious. Otherwise, there exists a chain whose length is $\operatorname{EAht}(a)$ and it ends in $(0: a)$. Let $(0: 1) \subset\left(0: a_{1}\right) \subset \cdots \subset$ $\left(0: a_{r-1}\right) \subset(0: a)$ be this chain. Moreover, we have $(0: a) \subset \cdots \subset\left(0: a^{n(a)-1}\right)$. So we obtain the following chain: $(0: 1) \subset\left(0: a_{1}\right) \subset \cdots \subset\left(0: a_{r-1}\right) \subset(0: a) \subset$ $\left(0: a^{2}\right) \subset \cdots \subset\left(0: a^{n(a)-1}\right)$ whose length is EAht $(a)+n(a)-2$. Consequently, $\operatorname{EAht}(a)+n(a)-2 \leq \operatorname{EAdim}(A)$.

Corollary 5. If $a \in \operatorname{nil}(A) \backslash\{0\}$ satisfies EAht $(a)=$ EAdim $(A)$ is finite then $n(a)=2$. In particular, if $\operatorname{EAdim}(A)=1$ then for all $a \in \operatorname{nil}(A) \backslash\{0\}, n(a)=2$.

Theorem 6. Let $A_{1}$ and $A_{2}$ be two rings; then;

(1) $\operatorname{EAdim}\left(A_{1} \times A_{2}\right)$ is finite if and only if $\operatorname{EAdim}\left(A_{1}\right)$ and $\operatorname{EAdim}\left(A_{2}\right)$ are finite.

(2) $\operatorname{EAdim}\left(A_{1} \times A_{2}\right)=\operatorname{EAdim}\left(A_{1}\right)+\operatorname{EAdim}\left(A_{2}\right)+1$.

Proof. Let $(a, b) \in A_{1} \times A_{2}$ be a nonzero zero-divisor. If $a$ and $b$ are nonzero then $(0:(a, b))_{A_{1} \times A_{2}}=(0: a)_{A_{1}} \times(0: b)_{A_{2}}$.

If one of them is zero, for example, $a=0$ then $(0$ : $(a, b))_{A_{1} \times A_{2}}=A_{1} \times(0: b)_{A_{2}}$.

(1) " $\Rightarrow$ " Let $\operatorname{EAdim}\left(A_{1} \times A_{2}\right)=n$, suppose that $\operatorname{EAdim}\left(A_{1}\right)$ or $\operatorname{EAdim}\left(A_{2}\right)$ is infinite; for example, EAdim $\left(A_{1}\right)$ is infinite. Then there exists $r \geq n+1$ and $(0: 1) \subset\left(0: a_{1}\right) \subset \cdots \subset\left(0: a_{r}\right)$ a chain in $A_{1}$; then $(0:(1,0)) \subset\left(0:\left(a_{1}, 0\right) \subset \cdots \subset\left(0:\left(a_{r}, 0\right)\right)\right.$ is a chain of elementary annihilators in $A_{1} \times A_{2}$ whose length is $r>n$, contradiction.

" $\Leftarrow$ " If we assume that $n=\operatorname{EAdim}\left(A_{1}\right)$, then there is a chain of length $n$ in $A_{1}$; let $(0: 1)_{A_{1}} \subset(0$ : $\left.a_{1}\right)_{A_{1}} \subset \cdots \subset\left(0: a_{n}\right)_{A_{1}}$ be this chain. In the same way we put $m=\operatorname{EAdim}\left(A_{2}\right)$ and we take $(0: 1)_{A_{2}} \subset$ $\left(0: b_{1}\right)_{A_{2}} \subset \cdots \subset\left(0: b_{m}\right)_{A_{2}}$ as a chain of length $m$. Then $(0:(1,1))_{A_{1} \times A_{2}} \subset\left(0:\left(a_{1}, 1\right)\right)_{A_{1} \times A_{2}} \subset \cdots \cdots$ $\left(0:\left(a_{n}, 1\right)\right)_{A_{1} \times A_{2}} \subset\left(0:\left(a_{n}, b_{1}\right)\right)_{A_{1} \times A_{2}} \subset \cdots \cdots$ $\left(0:\left(a_{n}, b_{m}\right)\right)_{A_{1} \times A_{2}} \subset\left(0:\left(0, b_{m}\right)\right)_{A_{1} \times A_{2}}$ is an elementary annihilator chain of $A_{1} \times A_{2}$ whose length is $n+m+1$ that is maximal, because of the inclusion $(0:(a, b))_{A_{1} \times A_{2}} \subset(0$ : $(c, d))_{A_{1} \times A_{2}} \Leftrightarrow(0: a)_{A_{1}} \subset(0: c)_{A_{1}}$ or $(0: b)_{A_{2}} \subset(0: d)_{A_{2}}$. Then $\operatorname{EAdim}\left(A_{1} \times A_{2}\right)$ is finite and $\operatorname{EAdim}\left(A_{1} \times A_{2}\right)=$ $n+m+1$.

(2) If $\operatorname{EAdim}\left(A_{1} \times A_{2}\right)$ is infinite (that is $\operatorname{EAdim}\left(A_{1}\right)$ or $\operatorname{EAdim}\left(A_{2}\right)$ is infinite, by (1)) then the result is obvious. The finite case is shown in the proof of (1) “ $\Leftarrow$ ".

By induction, we have the following result.

Corollary 7. (1) Let $A_{1}, \ldots, A_{r}$ be some rings, one has $\operatorname{EAdim}\left(A_{1} \times \cdots \times A_{r}\right)=\sum_{i=1}^{r} \operatorname{EAdim}\left(A_{i}\right)+r-1$.

(2) If $A$ is a domain and $n \in \mathbb{N}^{*}$ then $\operatorname{EAdim}\left(A^{n}\right)=n-1$.

\section{The EAdimension and the Zero-Divisor Graph}

Let $A$ be a ring. The zero-divisor graph of $A$ is defined to be the graph whose vertices are the nonzero zero-divisors of $A$ and its edges are the pairs $\{a, b\}$ satisfying $a b=0$. We denote this graph by $\Gamma(A)$. For the simplicity of writing we still denote by $\Gamma(A)$ the set of nonzero zero-divisors of $A$.

$\Gamma(A)$ is said to be connected if for every two different vertices $a$ and $b$ of $\Gamma(A)$ there is a sequence $a_{1}, \ldots, a_{n} \in$ $\Gamma(A)$ such as $a=a_{1}, b=a_{n}$ and $\left\{a_{i}, a_{i+1}\right\}$ is an edge, $\forall 1 \leq i \leq n-1$. This sequence is called a path connecting $a$ and $b$ with length $n-1$. $\Gamma(A)$ is said to be complete if each two distinct vertices form an edge. We call the distance between $a$ and $b$ the least length of a path connecting them, denoted by $d_{A}(a, b)$ or $d(a, b)$. We call the diameter of $\Gamma(A)$, denoted $\operatorname{diam}(\Gamma(A))$, the supremum of the set $\{d(a, b) ; a, b \in$ $\Gamma(A)\}$. In [1], Anderson and Livingston showed that $\Gamma(A)$ is connected and $\operatorname{diam}(\Gamma(A)) \in\{0,1,2,3\}$.

For an integer $r \geq 2$, Anderson and Livingston defined $\Gamma(A)$ to be $r$-partite complete if $\Gamma(R)=\Gamma_{1} \cup \cdots \cup \Gamma_{r}$, where the $\Gamma_{i}$ s are nonempty disjoined sets and for all $x \neq y$ in $\Gamma(A)$ satisfy $x y \neq 0$ if and only if there exists $1 \leq i \leq r$ such that $x, y \in \Gamma_{i}$. In this paper we extend the definition of $\mathrm{r}$-partite complete graph to the case when $r$ is infinite.

Lemma 8 (see [2], Theorem 6). (1) If $(0: a)$ is an elementary annihilator that is maximal (in the set of proper annihilators of A) then it is prime.

(2) Let $a, b \in A \backslash\{0\}$; if $(0: a)$ is maximal and $(0: b) \nsubseteq$ $(0: a)$ then $b \in(0: a)$.

Proposition 9. Let $A$ be a reduced ring that is not a domain and $a \neq b$ be two nonzero zero-divisors such that $(0: a)$ and $(0: b)$ are maximal; then $a b \neq 0$ if and only if $(0: a)=(0: b)$.

Proof. “ $\Rightarrow$ ” Immediately, by Lemma 8 “ $\Leftarrow$ ” If $(0: a)=(0: b)$, suppose that $b a=0 . b a=0 \Rightarrow b \in(0: a)=(0: b) \Rightarrow b^{2}=$ 0 , contradiction. Then $a b \neq 0$.

Theorem 10. If $A$ is a nonreduced ring then $\operatorname{EAdim}(A)=1$ if and only if $\Gamma(A)$ is complete.

Proof. " $\Leftarrow$ " if $\Gamma(A)$ is complete then, according to [1, Theorem 2.8], we have for all $x, y \in Z(A), x y=0$. Then $Z(A)=(0: c)$, $\forall c \in Z(A) \backslash\{0\}$. So all nonzero elementary annihilators are equal, and then $\operatorname{EAdim}(A)=1$.

“ $\Rightarrow$ " Let $a \in \operatorname{nil}(A) \backslash\{0\}$; then $a^{2}=0$, according to Corollary 5. If $Z(A)=\{0, a\}$ then $\Gamma(A)$ is complete. Otherwise, for all $b \in Z(A) \backslash\{0, a\}$ we have $(0: a)$ and $(0: b)$ are maximal. Suppose that $(0: a) \neq(0: b)$, according to Lemma $8(2), a b=0$ and there, for example, $y \in(0: a) \backslash(0$ : $b)$; then $(0: b) \subset(0: y b)$, contradiction to the maximality of $(0: b)$. Then $(0: a)=(0: b)$ and $a b=0$. Consequently, $Z(A)=\operatorname{nil}(A)$ and all nonzero zero-divisors $b$ satisfy $b^{2}=0$. It follows that for all $a, b \in Z(A), a b=0$. According to [1, Theorem 2.8], $\Gamma(A)$ is complete.

Theorem 11. If $\Gamma(A)=\bigcup_{i \in I} \Gamma_{i}$ is r-partite complete graph with $r=\operatorname{card}(I) \in \mathbb{N} \cup\{\infty\}, r \geq 2$ then $r=2$ under one of the following conditions:

(1) two of the $\Gamma_{i}$ 's contain, each one, more than one element;

(2) A is reduced. 
Proof. Suppose that $r \geq 3$.

First case: If two among the $\Gamma_{i}$ 's contain, each one, more then one element. Assume that $\Gamma(A)=\Gamma_{1} \cup \Gamma_{2} \cup \Gamma_{3} \cup \cdots$, where $\Gamma_{1}$ and $\Gamma_{2}$ have, each one, at least two elements. Let $a_{i} \in \Gamma_{i}, 1 \leq i \leq 3$ and $a_{i} \neq b_{i} \in \Gamma_{i}, 1 \leq i \leq 2$. We have $\left(a_{1}+a_{2}\right) a_{3}=0$; then $a_{1}+a_{2}$ is a divisor of zero in $A$. Suppose that $a_{1}+a_{2}=0$; then $b_{1}\left(a_{1}+a_{2}\right)=0 \Rightarrow b_{1} a_{1}=0$, contradiction. Then $a_{1}+a_{2} \in \Gamma(A)$. Suppose that $a_{1}+a_{2} \in \Gamma_{3} \cup \cdots$; then $b_{1}\left(a_{1}+a_{2}\right)=0 \Rightarrow b_{1} a_{1}=0$, contradiction; then $a_{1}+a_{2} \in \Gamma_{1} \cup \Gamma_{2}$. If $a_{1}+a_{2} \in \Gamma_{1}$ then $b_{2}\left(a_{1}+a_{2}\right)=0 \Rightarrow b_{2} a_{2}=0$, contradiction. Then $a_{1}+a_{2} \in \Gamma_{2}$ and $b_{1}\left(a_{1}+a_{2}\right)=0 \Rightarrow b_{1} a_{1}=0$, contradiction.

Second Case: If A Is Reduced. Assume that $\Gamma(A)=\Gamma_{1} \cup \Gamma_{2} \cup$ $\Gamma_{3} \cup \cdots$. Let $a_{i} \in \Gamma_{i}, 1 \leq i \leq 3$, we have $\left(a_{1}+a_{2}\right) a_{3}=0$ then $a_{1}+a_{2}$ is a divisor of zero in $A$. Suppose that $a_{1}+a_{2}=0$, then $a_{1}\left(a_{1}+a_{2}\right)=0 \Rightarrow a_{1}^{2}=0$, contradiction. Then $a_{1}+a_{2} \in \Gamma(A)$. Suppose that $a_{1}+a_{2} \in \Gamma_{3} \cup \cdots$ then $a_{1}\left(a_{1}+a_{2}\right)=0 \Rightarrow a_{1}^{2}=0$, contradiction. Then $a_{1}+a_{2} \in \Gamma_{1} \cup \Gamma_{2}$. If $a_{1}+a_{2} \in \Gamma_{1}$ then $a_{2}\left(a_{1}+a_{2}\right)=0 \Rightarrow a_{2}^{2}=0$, contradiction. Then $a_{1}+a_{2} \in \Gamma_{2}$ and then $a_{1}\left(a_{1}+a_{2}\right)=0 \Rightarrow a_{1}^{2}=0$, contradiction. We conclude that $r=2$.

Theorem 12. If $A$ is reduced then $\operatorname{EAdim}(A)=1$ if and only if $\Gamma(A)$ is bipartite complete.

Proof. " $\Rightarrow$ " In $\Gamma(A)$, we define the relation “ $\sim$ " by the following: $x \sim y$ if $(0: x)=(0: y) . \sim$ is a relation of equivalence. For all $x \in \Gamma(A)$, we denote by $\Gamma_{x}$ its equivalence class. The different classes $\Gamma_{x}$ form a partition of $\Gamma(A)$ and we write $\Gamma(A)=\bigcup_{i \in I} \Gamma_{x_{i}}$. Since $A$ is reduced and not a domain, then there exist nonzero elements $b \neq a$ satisfying $b a=0$. Now, $\operatorname{EAdim}(A)=1$; then $(0: a)$ and $(0: b)$ are maximal. According to Proposition $9,(0: a) \neq(0: b)$; then $\Gamma_{a} \neq \Gamma_{b}$; then $r=\operatorname{card}(I) \geq 2$.

If $y \neq z \in \Gamma_{x}$ then $(0: z)=(0: y)$ then $y z \neq 0$, by Proposition 9.

Let $\Gamma_{x} \neq \Gamma_{y}$; then $(0: x) \neq(0: y)$; then, by Lemma $8, x y=$ 0 . And we conclude that $\Gamma(A)$ is $r$-partite complete. According to Theorem $11, \Gamma(A)$ is bipartite complete.

" $\Leftarrow$ " Assume that $\Gamma(A)=\Gamma_{1} \cup \Gamma_{2}$ is bipartite complete. If $a \in \Gamma_{i}$ then $(0: a)=\Gamma_{j} \cup\{0\}$, for $i \neq j \in\{1,2\}$. Let $a, b \in \Gamma(A)$, if $a, b \in \Gamma_{i}$ then $(0: a)=(0: b)=\Gamma_{j} \cup\{0\}$. Otherwise $(0: a)$ and $(0: b)$ are incomparable. Then for all $a \in \Gamma(A)$, $\operatorname{EAht}(a)=1$. It follows that $\operatorname{EAdim}(A)=1$.

Theorem 13. Let $R$ be a ring.

(1) If $\operatorname{EAdim}(R)=1$ then $\operatorname{diam}(R) \leq 2$.

(2) If $\operatorname{diam}(R) \leq 1$ or $\operatorname{diam}(R)=2$ and $R$ is reduced then $\operatorname{EAdim}(R)=1$.

(3) If $\operatorname{diam}(R)=2$ and $R$ is not reduced or diam $(R)=3$ then $\operatorname{EAdim}(R) \geq 2$.

Proof. (1) If $R$ is reduced then, by Theorem $12, \Gamma(R)$ is bipartite complete; then $\operatorname{diam}(R) \leq 2$.

If $R$ is not reduced then, by Theorem $10, \Gamma(R)$ is complete then, by Theorem 2.8 of [1], for all $x, y \in Z(R), x y=0$. Then, by Theorem 2.6 of [3], $\operatorname{diam}(R) \leq 1$.
(2) If $\operatorname{diam}(R)=2$ and $R$ is reduced then, by Theorem 2.6 of [3], $R$ is reduced with exactly two minimal primes and at least three nonzero zero-divisors. Then $Z(R)=P_{1} \cup P_{2}$, where $P_{1}, P_{2}$ are the two minimal primes of $R$; they satisfy $P_{1} \cap P_{2}=(0)$. Then for all $p_{1} \in P_{1}$ and $p_{2} \in P_{2}, p_{1}, p_{2}=0$ and for $x \neq y \in P_{i} \backslash\{0\}, x y \neq 0$. Consequently, $\Gamma(R)=\left[P_{1} \backslash\right.$ $\{0\}] \cup\left[P_{2} \backslash\{0\}\right]$ is bipartite complete graph. According to Theorem 12, $\operatorname{EAdim}(R)=1$.

If $\operatorname{diam}(R) \leq 1$ : if $\operatorname{diam}(R)=0$, by Theorem 2.6 of [3], $R$ is isomorphic to either $\mathbb{Z}_{4}$ or $\mathbb{Z}_{2}[y] /\left(y^{2}\right)$ and in both cases $R$ has a unique nonzero elementary annihilator then $\operatorname{EAdim}(R)=$ 1.

Now, if $\operatorname{diam}(R)=1$, using Theorem 2.6 of [3], $x y=0$ for each distinct pair of zero-divisors and $R$ has at least two nonzero zero-divisors. According to Theorem 2.8 of [1], $\Gamma(R)$ is complete and $R$ is not reduced. By Theorem $10, \operatorname{EAdim}(R)=$ 1.

(3) If $\operatorname{diam}(R)=3$ then, by (1), $\operatorname{EAdim}(R) \neq 1$. Since $\operatorname{diam}(R)=3$ then $R$ is not a domain then $\operatorname{EAdim}(R) \neq 0$. Consequently, $\operatorname{EAdim}(R) \geq 2$. If $\operatorname{diam}(R)=2$ and $R$ is not reduced: $\operatorname{diam}(R)=3$ then $R$ is not a domain then $\operatorname{EAdim}(R) \neq 0$. Suppose that $\operatorname{EAdim}(R)=1$; then by Theorem $10, \Gamma(R)$ is complete then $\operatorname{diam}(R)=1$, contradiction. Then $\operatorname{EAdim}(R) \geq 2$.

Lemma 14. Let $A$ be a ring, and $F=\left\{P_{1}, \ldots, P_{r}\right\}, r>1$ a set of distinct prime ideals which are incomparable. For a an element of $R$ we denote $E(a)=\{P \in F ; a \notin P\}$. Let $R=A /\left(P_{1} \cap \cdots \cap P_{r}\right)$.

(1) For all subsets $E$ of $F$ there is $a \in A$ such that $E(a)=E$.

(2) Let $a \in A, \bar{a} \in Z(R) \Leftrightarrow E(a) \neq F$. In particular, $\bar{a}=$ $\overline{0} \Leftrightarrow E(a)=\emptyset$.

(3) Let $E_{1}$ and $E_{2}$ be two nonempty subsets of $F$. If $\bigcap_{P \in E_{1}} P \subseteq \bigcap_{P \in E_{2}} P$ then $E_{2} \subseteq E_{1}$.

(4) Let $a, b \in A . \bar{b} \in(\overline{0}: \bar{a}) \Leftrightarrow b \in \bigcap_{P \in E(a)} P$.

(5) The map $\varphi:\{(\overline{0}: \bar{a}), \bar{a} \neq \overline{0}\} \rightarrow \mathscr{P}(F) \backslash\{\emptyset\},(\overline{0}: \bar{a})$ $\longmapsto E(a)$ is a decreasing bijection, here $\mathscr{P}(F)$ denotes the set of $F$ subsets.

Proof. (1) If $E=\emptyset$, take $a=0$ then $a \in P$, for all $P \in F$ then $E(a)=\emptyset$ and the result is true in this case.

If $E=F$, take $a=1$ then $a \notin P$, for all $P \in F$ then $E(a)=F$

Now if $E \notin\{F, \emptyset\}: E(a)=E \Leftrightarrow a \in P, \forall P \notin E$ and $a \notin P, \forall P \in E \Leftrightarrow a \in\left(\bigcap_{P \in F \backslash E} P\right) \backslash\left(\bigcup_{P \in E} P\right)$. Suppose that $\left(\bigcap_{P \in F \backslash E} P\right) \backslash\left(\bigcup_{P \in E} P\right)=\emptyset \Rightarrow \bigcap_{P \in F \backslash E} P \subseteq \bigcup_{P \in E} P$; then there exists $P_{i_{0}} \in E$ such that $\bigcap_{P \in F \backslash E} P \subseteq P_{i_{0}}$; then there exists $P_{i} \in$ $F \backslash E$ such that $P_{i_{1}} \subseteq P_{i_{0}}$. Since the $P$ 's in $F$ are incomparable under inclusion then $P_{i_{1}}=P_{i_{0}}$, then $P_{i_{0}} \in(F \backslash E) \cap E=$ $\emptyset$, contradiction. Consequently, there exists $a \in A$ such that $E(a)=E$.

(2) Let $a \in A, \bar{a} \in Z(R) \Leftrightarrow \exists \bar{b} \neq \overline{0} / \bar{a} \bar{b}=\overline{0} \Leftrightarrow \exists b \in$ $A / b \notin \bigcap_{P \in F} P$ and $b a \in \bigcap_{P \in F} P \Leftrightarrow \exists b \in A / E(b) \neq \emptyset$ and $b a \in P, \forall P \in E(a) \Leftrightarrow \exists b \in A / E(b) \neq \emptyset$ and $b \in P, \forall P \in$ $E(a) \Leftrightarrow \exists b \in A / E(b) \neq \emptyset$ and $E(a) \subseteq F \backslash E(b) \Leftrightarrow E(a) \neq F$. In the last equivalence the indirect sense " $\Leftarrow$ " is obtained by (1). 
(3) Suppose that $E_{1}=\left\{Q_{1}, \ldots, Q_{s}\right\}$ and $E_{2}=\left\{R_{1}, \ldots, R_{t}\right\}$, where the $Q_{i}$ 's (resp., $P_{i}$ 's) are pairwise different. $Q_{1} \cap \cdots \cap Q_{s} \subseteq$ $R_{1} \cap \cdots \cap R_{t} \Rightarrow Q_{1} \cap \cdots \cap Q_{s} \subseteq R_{1}$ then one of the $Q_{i}$ 's is contained in $R_{1}$; for example, $Q_{1} \subseteq R_{1}$. Since the elements of $F$ are incomparable under inclusion then $R_{1}=Q_{1}$.

$Q_{1} \cap \cdots \cap Q_{s} \subseteq R_{1} \cap \cdots \cap R_{t} \Rightarrow Q_{1} \cap \cdots \cap Q_{s} \subseteq R_{2}$ then one of the $Q_{i}$ 's is contained in $R_{2}$. Suppose that $Q_{1} \subseteq R_{2}$; then $Q_{1}=R_{2}$ then $R_{1}=R_{2}$, contradiction. Then there exists $i \geq 2$ such that $Q_{i} \subseteq R_{2}$; for example, $Q_{2} \subseteq R_{2}$; then $R_{2}=Q_{2}$.

We repeat this process until reaching the stage number $n=\min (s, t)$.

Suppose that $s<t$; then $Q_{1} \cap \cdots \cap Q_{s}=R_{1} \cap \cdots \cap R_{s} \subseteq$ $R_{s+1}$; then there exists $k \leq s$ such that $R_{k} \subseteq R_{s+1}$ that is, $R_{k}=R_{s+1}$, contradiction. Consequently, $s \geq t$ and we get $E_{1}=\left\{R_{1}, \ldots, R_{t}, Q_{t+1}, \ldots, Q_{s}\right\}$; then $E_{2} \subseteq E_{1}$.

(4) Let $a, b \in A, \bar{b} \in(\overline{0}: \bar{a}) \Leftrightarrow b a \in \bigcap_{P \in F} P \Leftrightarrow b \in$ $\bigcap_{P \in E(a)} P$.

(5) We check that $\varphi$ is well defined: put $(\overline{0}: \bar{a})=(\overline{0}$ : $\bar{b})$. According to $(4), \bar{x} \in(\overline{0}: \bar{a}) \Leftrightarrow x \in \bigcap_{P \in E(a)} P$. Then $x \in \bigcap_{P \in E(a)} P \Leftrightarrow x \in \bigcap_{P \in E(b)} P$; then $\bigcap_{P \in E(b)} P=\bigcap_{P \in E(a)} P$. According to (3), $E(b)=E(a)$.

For $\bar{a} \neq \overline{0}, E(a) \neq \emptyset$; then $E(a) \in \mathscr{P}(F) \backslash\{\emptyset\}$. Then $\varphi$ is well defined.

$\varphi$ is injective, by (4).

We show that $\varphi$ is surjective: let $E \in \mathscr{P}(F) \backslash\{\emptyset\}$. According to (1), there exists $a \in A$ such that $E(a)=E$. Since $E \neq \emptyset$ then $\bar{a} \neq \overline{0}$ and $\varphi(\overline{0}: \bar{a})=E$.

$(\overline{0}: \bar{a}) \subseteq(\overline{0}: \bar{b}) \Rightarrow \bigcap_{P \in E(a)} P \subseteq \bigcap_{P \in E(b)} P$. Then, according to (3), $E(b) \subseteq E(a)$. Thus $\varphi$ is a decreasing bijection.

Theorem 15. Let $A$ be a ring and $P_{1}, \ldots, P_{r}, r \geq 1$ be different incomparable prime ideals of $A$. Then $\operatorname{EAdim}\left(A /\left(P_{1} \cap \cdots \cap\right.\right.$ $\left.\left.P_{r}\right)\right)=r-1$.

Proof. If $r=1$ then $A / P_{1}$ is a domain and the result is checked.

Now let $r \geq 2 .\left\{P_{1}\right\} \subset\left\{P_{1}, P_{2}\right\} \subset \cdots \subset\left\{P_{1}, \ldots\right.$, $\left.P_{r-1}\right\} \subset\left\{P_{1}, \ldots, P_{r}\right\}$ is decreasing sequence in $\mathscr{P}(F) \backslash\{\emptyset\}$ with maximal length. Using the bijection $\varphi$ defined in Lemma 14, $\varphi^{-1}\left(\left\{P_{1}, \ldots, P_{r}\right\}\right) \subset \varphi^{-1}\left(\left\{P_{1}, \ldots, P_{r-1}\right\}\right) \quad \subset \quad \cdots \quad \subset$ $\varphi^{-1}\left(\left\{P_{1}, P_{2}\right\}\right) \subset \varphi^{-1}\left(\left\{P_{1}\right\}\right)$ is a chain of elementary annihilators in $A /\left(P_{1} \cap \cdots \cap P_{r}\right)$ that has a maximal length. Then $\operatorname{EAdim}\left(A /\left(P_{1} \cap \cdots \cap P_{r}\right)\right)=r-1$.

Example 16. A ring is said to be semilocal ring if it has a finite number of maximal ideals. Let $A$ be a semilocal ring with $n$ maximal ideal; then EAdim $(A / J)=n-1$, where $J$ is the Jacobson radical of $A$. We obtain this result by using the previous theorem.

A ring is called a noetherian spectrum ring if it satisfies the ascending chain condition (acc) on radical ideals; equivalently each radical ideal is a radical of finitely generated ideal. The set of prime ideals of a ring $A$ which are minimal over an ideal $I$, denoted by $\min _{I}(A)$, is finite in the case when $A$ is a noetherian spectrum ring. If $I=(0)$, we denote by $\min (A)$ instead of $\min _{I}(A)$. For more information about noetherian spectrum rings see [4, Chapter 2].

Proposition 17. Let $A$ be a noetherian spectrum ring, for all ideals $I, \operatorname{EAdim}(A / \sqrt{I})=\left|\min _{I}(A)\right|-1$.

Proof. Since $A$ is a noetherian spectrum ring then $\min _{I}(A)$ is finite ([4], Chapter 2, Corollary 2.1.10). Assume that $\min _{I}(A)=\left\{P_{1}, \ldots, P_{r}\right\}, r=\left|\min _{I}(A)\right| \in \mathbb{N}^{*}$. The $P_{i}$ 's are incomparable, then we get the result by using Theorem 15.

Definition 18 (according to [5]). Let $R$ be a ring.

(1) One calls the chromatic number of $R$ the minimal number of colors used to color the elements of $R$ such that each two adjacent elements (with zero product) have different colors, denoted by $\chi(R)$.

(2) One says that the ring $R$ is a coloring if its chromatic number is finite.

Theorem 19. If $R$ is a reduced coloring then $\operatorname{EAdim}(R)=$ $\chi(R)-2$.

Proof. If $R$ is a reduced coloring, according to [5, Theorem 3.8], $\min (R)$ is finite. And if $|\min (R)|=n$ then $\chi(R)=n+$ 1. Let $\min (R)=\left\{P_{1}, \ldots, P_{n}\right\}$; then $R=R /\left(P_{1} \cap \cdots \cap P_{n}\right)$. According to Theorem 15, $\operatorname{EAdim}(R)=n-1=\chi(R)-2$.

Let $A$ be a ring such that $\operatorname{EAdim}(A)=n \geq 1$. For all vertices $a$ in $\Gamma(A)$, we denote $i(a)=\operatorname{EAht}(a)$ and $j(a)=$ $\max \{\operatorname{EAht}(b) ;(0: a) \subseteq(0: b)\}$. For all $i \leq j \leq n$, we denote by $\Gamma_{i, j}(A)$ the subgraph of $\Gamma(A)$ whose vertices form the following set $\{a ; i(a)=i$ et $j(a)=j\}$.

Theoretically we can write $\Gamma(A)=\bigcup_{1 \leq i \leq j \leq n} \Gamma_{i, j}(A)$.

Remark 20. Let $A$ be a ring such that $\operatorname{EAdim}(A)=n \geq 2$. If $\Gamma_{1,1}(A) \neq \emptyset$ then $\operatorname{diam}(\Gamma(A))=2$. Indeed, consider $a \in \Gamma_{1,1}(A)$ and $b \in \Gamma(A) \backslash \Gamma_{1,1}(A)$. There exists $i \geq 1$ and $j \geq 2$ such that $b \in \Gamma_{i, j}(A)$. Suppose that $a b \neq 0$ then $b \notin(0: a)$. According to Lemma $8(2)(0: b) \subseteq(0: a)$, this contradicts the fact that $a \in \Gamma_{1,1}(A)$. Then $a b=0$ and $\{a, b\}$ is an edge. Now take $x \neq y \in \Gamma(A)$, three cases are possible. If $x, y \in \Gamma_{1,1}(A)$ then take $b \in \Gamma(A) \backslash \Gamma_{1,1}(A)$ and the chain $x-b-y$ is of length 2 then $d(x, y) \leq 2$. If only $x$ is in $\Gamma_{1,1}(A)$ then $x-y$ is an edge. If $x, y \in \Gamma(A) \backslash \Gamma_{1,1}(A)$ then take $a \in \Gamma_{1,1}(A)$ and $x-a-y$ is a chain of length 2 . Then, in all cases, $d(x, y) \leq 2$ that is $\operatorname{diam}(\Gamma(A)) \leq 2$. Now $\operatorname{EAdim}(A)=n \geq 2$, then there exists $a, b$ such that $(0: 1) \subset(0: a) \subset(0: b)$. Let $x \in(0: b) \backslash(0: a)$ then $x a \neq 0$ then $d(x, a) \geq 2$. Consequently, $\operatorname{diam}(\Gamma(A))=2$.

\section{Isometric Maximal Elementary Annihilator Rings}

Definition 21. Let $R$ be a ring with finite EA dimension; one says that $R$ is an isometric maximal elementary annihilator ring, in short an IMEA-ring if its all maximal elementary annihilators have the same height.

Example 22. The ring $\mathbb{Z} / 8 \mathbb{Z}$ is an IMEA-ring. Indeed, the elementary annihilators of $\mathbb{Z} / 8 \mathbb{Z}$ are $(\overline{0}: \overline{1}),(\overline{0}: \overline{2}),(\overline{0}: \overline{4})$ 
and $(\overline{0}: \overline{6})$. They satisfy $(\overline{0}: \overline{1}) \subset(\overline{0}: \overline{2}) \subset(\overline{0}: \overline{4})$ and $(\overline{0}: \overline{1}) \subset(\overline{0}: \overline{2}) \subset(\overline{0}: \overline{6})$.

Theorem 23. Let $R_{1}$ and $R_{2}$ be two rings; then $R_{1} \times R_{2}$ is an IMEA-ring if and only if $R_{1}$ and $R_{2}$ are two IMEA-rings.

Proof. By Theorem 6(1), EAdim $\left(R_{1} \times R_{2}\right)$ is finite if and only if $\operatorname{EAdim}\left(R_{1}\right)$ and $\operatorname{EAdim}\left(R_{2}\right)$ are finite.

$(0:(a, b))$ is a maximal elementary annihilator in $R_{1} \times R_{2}$ if and only if $(0:(a, b))=R_{1} \times(0: b)$, and $(0: b)$ is a maximal elementary annihilator in $R_{2}$ or $(0:(a, b))=(0: a) \times R_{2}$, and $(0: a)$ is a maximal elementary annihilator in $R_{1}$, then all maximal elementary annihilators of $R_{1} \times R_{2}$ have the same hight if and only if all maximal elementary annihilators of $R_{1}$ have the same height and also for the maximal elementary anniWlitorthof fRllowing result, inductively.

Corollary 24. Let $R_{1}, \ldots, R_{n}$ be some rings. We have $R_{1} \times R_{2} \times$ $\cdots \times R_{n}$ is an IMEA-ring if and only if each $R_{i}$ is an IMEA-ring.

Let $R$ be a domain, we say that $R$ is atomic if each nonzero nonunit element of $R$ decomposes into a finite product of irreducibles, according to [6]. An atomic domain is called a half factorial domain, in short a HFD if $x_{1} \cdots x_{n}=y_{1} \cdots y_{m}$ are two decompositions into irreducibles then $n=m$. This concept was introduced by Zaks in [7]. A HFD is called a unique factorization domain, in short a UFD if $x_{1} \cdots x_{n}=$ $y_{1} \cdots y_{n}$ are two decompositions into irreducibles then the $x_{i}$ 's and the $y_{i}$ 's are associates after reordering them. It is well known that a UFD is an atomic domain in which each irreducible is primed by $[8$, Theorem 1$]$.

Proposition 25. If $R$ is a UFD, then for all nonzero nonunit $a$ of $R$ we have $R /(a)$ is an IMEA-ring. Moreover if $a=$ $p_{1}^{m_{1}} \cdots p_{r}^{m_{r}}$ is the decomposition of a into prime elements then $\operatorname{EAdim}(R /(a))=m_{1}+\cdots+m_{r}-1$.

Proof. Let $a=p_{1}^{m_{1}} \cdots p_{r}^{m_{r}} \in R$. Suppose that $x \in R$; then $\bar{x} \in Z(R /(a)) \backslash\{\overline{0}\}$

$$
\begin{aligned}
& \Longleftrightarrow\left\{\begin{array}{l}
\bar{x} \neq \overline{0} \\
\exists \bar{y} \neq \overline{0} \\
\overline{x y}=\overline{0}
\end{array}\right.
\end{aligned}
$$

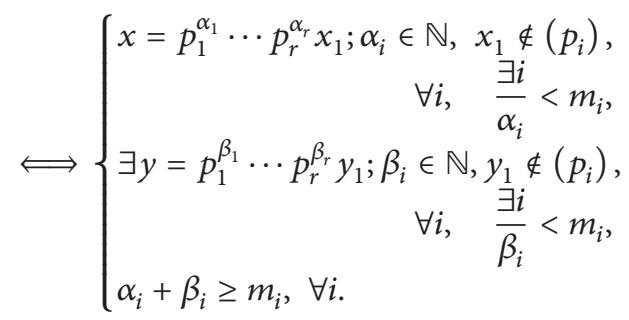

Then

$$
\begin{aligned}
Z\left(\frac{R}{(a)}\right)= & \left\{\overline{p_{1}^{\alpha_{1}} \cdots p_{r}^{\alpha_{r}} x_{1}} ;\left(\alpha_{1}, \ldots, \alpha_{r}\right) \in \mathbb{N}^{r} \backslash\{(0, \ldots, 0)\},\right. \\
& \left.x_{1} \in R \backslash \bigcup_{i}\left(p_{i}\right)\right\} .
\end{aligned}
$$

And for $\bar{x}=\overline{p_{1}^{\alpha_{1}} \cdots p_{r}^{\alpha_{r}} x_{1}} \neq \overline{0}$ (one among the $\alpha_{i}$ 's is $<m_{i}$ and $\left.x_{1} \in R \backslash \cup_{i}\left(p_{i}\right)\right)$, we have the following:

$$
\begin{gathered}
(\overline{0}: \bar{x})=\left\{\overline{p_{1}^{\beta_{1}} \cdots p_{r}^{\beta_{r}} y_{1}} ;\left(\beta_{1}, \ldots \beta_{r}\right) \in \mathbb{N}^{r} \backslash\{(0, \ldots, 0)\} / \beta_{i}\right. \\
\left.\geq m_{i}-\alpha_{i}, \forall i, y_{1} \in R \backslash \bigcup_{i}\left(p_{i}\right)\right\} .
\end{gathered}
$$

It is easy to check that the set of all elementary annihilators of $R /(a)$ is

$$
\begin{aligned}
& \left\{\left(\overline{0}: \overline{p_{1}^{\alpha_{1}} \cdots p_{r}^{\alpha_{r}}}\right) ;\right. \\
& \left.\quad\left(\alpha_{1}, \ldots, \alpha_{r}\right) \in\left[\left[0, m_{1}\right]\right] \times \cdots \times\left[\left[0, m_{r}\right]\right] \backslash\left(m_{1}, \ldots, m_{r}\right)\right\} .
\end{aligned}
$$

The maximal ones among them are

$$
\begin{aligned}
& \left(\overline{0}: \overline{p_{1}^{m_{1}-1} p_{2}^{m_{2}} \cdots p_{r}^{m_{r}}}\right),\left(\overline{0}: \overline{p_{1}^{m_{1}} p_{2}^{m_{2}-1} p_{3}^{m_{3}} \cdots p_{r}^{m_{r}}}\right), \\
& \ldots,\left(\overline{0}: \overline{p_{1}^{m_{1}} p_{2}^{m_{2}} \cdots p_{r}^{m_{r}-1}}\right) .
\end{aligned}
$$

A longest chain ending in one of them, for example, $(\overline{0}: \overline{1}) \subset$ $\left(\overline{0}: \overline{p_{1}}\right) \subset \cdots \subset\left(\overline{0}: \overline{p_{1}^{m_{1}-1}}\right) \subset\left(\overline{0}: \overline{p_{1}^{m_{1}-1} p_{2}}\right) \subset \cdots \subset(\overline{0}:$ $\left.\overline{p_{1}^{m_{1}-1} p_{2}^{m_{2}}}\right) \subset \cdots \subset\left(\overline{0}: \overline{p_{1}^{m_{1}} p_{2}^{m_{2}} \cdots p_{r-1}^{m_{r}-1} p_{r}}\right) \subset \cdots \subset(\overline{0}:$ $\left.\overline{p_{1}^{m_{1}-1} p_{2}^{m_{2}} \cdots p_{r}^{m_{r}}}\right)$, has the length $m_{1}+\cdots+m_{r}-1$. Thus all maximal elementary annihilators have the same height $m_{1}+$ $\cdots+m_{r}-1$ then $R /(a)$ is an IMEA-ring and $\operatorname{EAdim}(R /(a))=$ $m_{1}+\cdots+m_{r}-1$.

Proposition 26. Let $R$ be a HFD. $R$ is a UFD if and only if $\operatorname{EAdim}(R /(a))=\lambda(a)-1$, for all nonzero nonunit $a$. Where $\lambda(a)$ is the number of factors in a decomposition of a into irreducibles (counted with multiplicities).

Proof. " $\Rightarrow$ " is due to the previous proposition.

" $\Leftarrow$ " Let $a$ be an irreducible of $R$ then $\lambda(a)=1$; then $\operatorname{EAdim}(R /(a))=\lambda(a)-1=0$. According to Remark 3, $R /(a)$ is a domain; then $(a)$ is prime. According to [8, Theorem 1$]$, $R$ is a $U F D$.

Question 1. Are all finite EAdimensional rings IMEA-rings?

Question 2. Are all finite rings IMEA-rings?

\section{References}

[1] D. F. Anderson and P. S. Livingston, "The zero-divisor graph of a commutative ring," Journal of Algebra, vol. 217, no. 2, pp. 434447, 1999.

[2] I. Kaplansky, Commutative Rings, The University of Chicago Press, Chicago, Ill, USA, Revised edition, 1974.

[3] T. G. Lucas, "The diameter of a zero divisor graph," Journal of Algebra, vol. 301, no. 1, pp. 174-193, 2006. 
[4] A. Benhissi, Les Anneaux de Séries Formelles, vol. 124 of Queen's Papers in Pure and Applied Mathematics, Kingston, Ontario, Canada, 2003.

[5] I. Beck, "Coloring of commutative rings," Journal of Algebra, vol. 116, no. 1, pp. 208-226, 1988.

[6] P. M. Cohn, "Bezout rings and their subrings," Mathematical Proceedings of the Cambridge Philosophical Society, vol. 64, pp. 251-264, 1968.

[7] A. Zaks, "Half factorial domains," Bulletin of the American Mathematical Society, vol. 82, no. 5, pp. 721-724, 1976.

[8] P. M. Cohn, "Unique factorization domains," The American Mathematical Monthly, vol. 80, no. 1, pp. 1-18, 1973. 


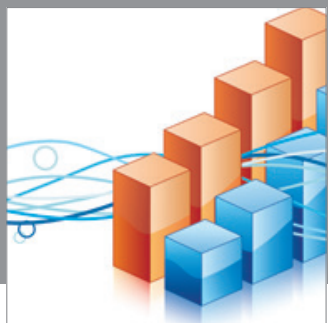

Advances in

Operations Research

mansans

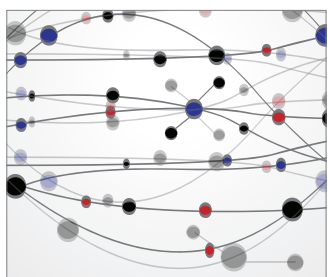

The Scientific World Journal
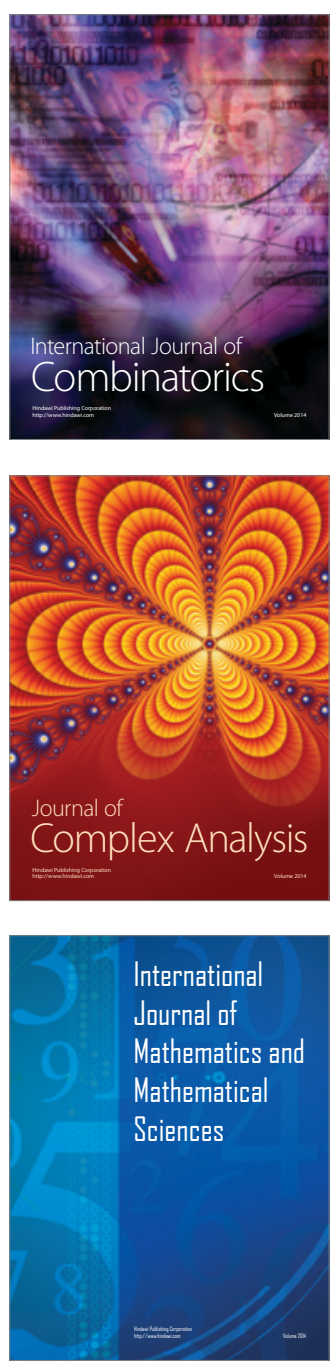
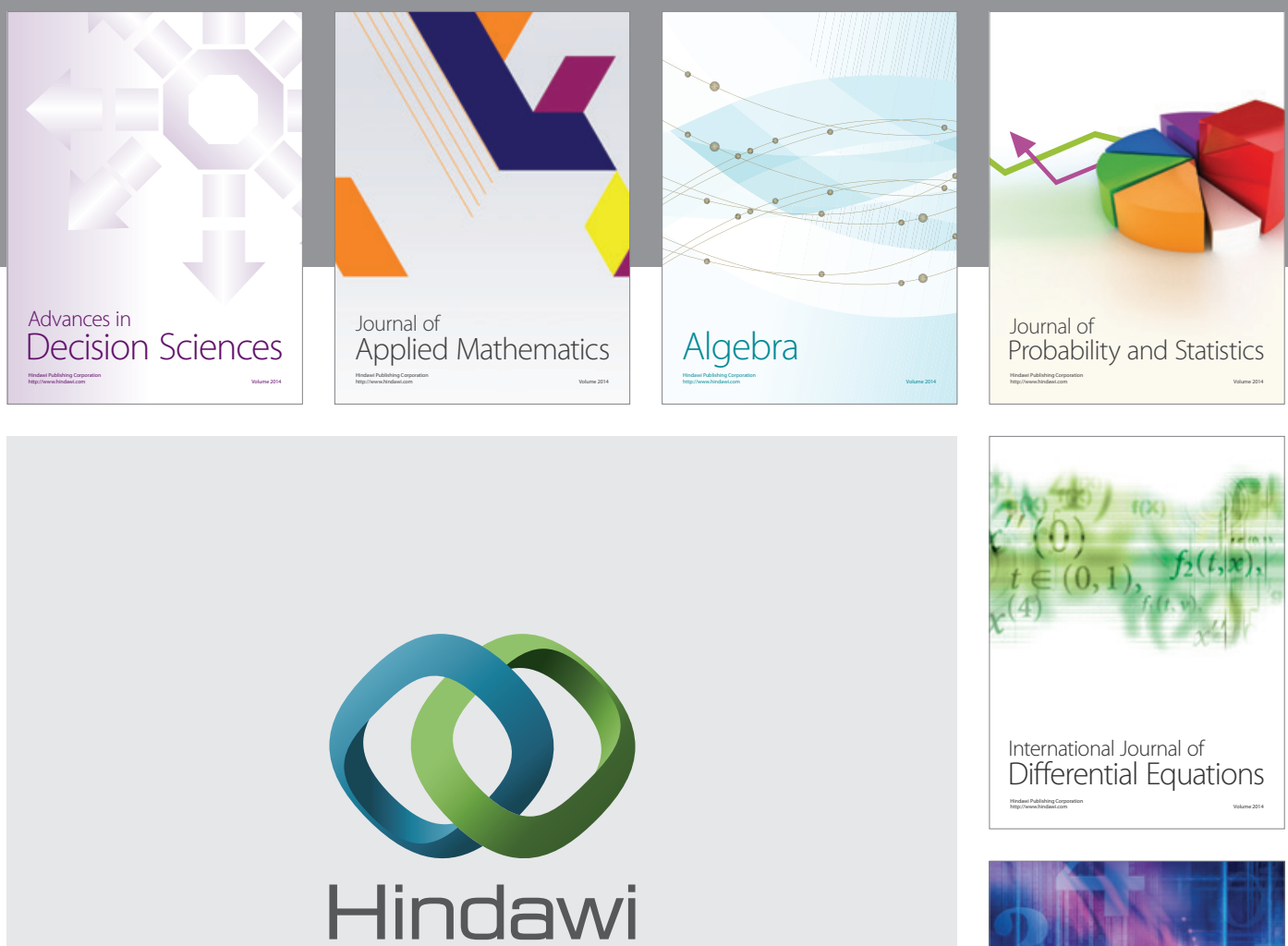

Submit your manuscripts at http://www.hindawi.com
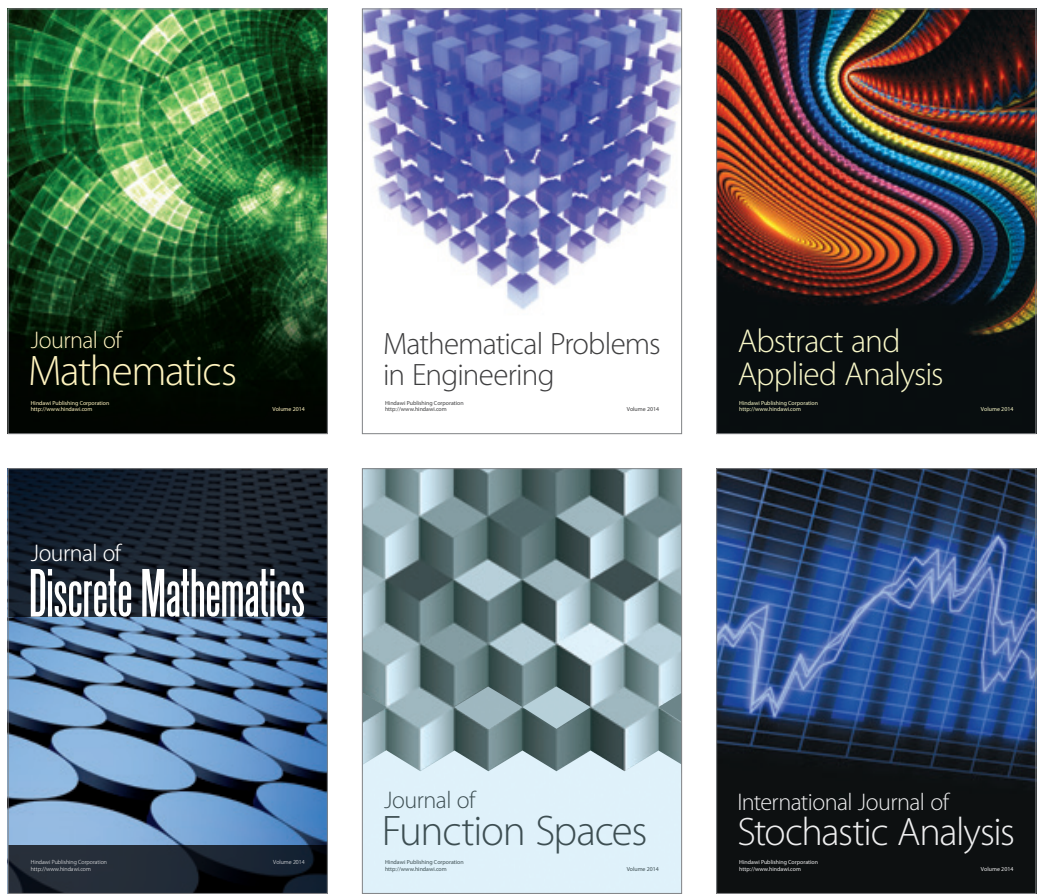

Journal of

Function Spaces

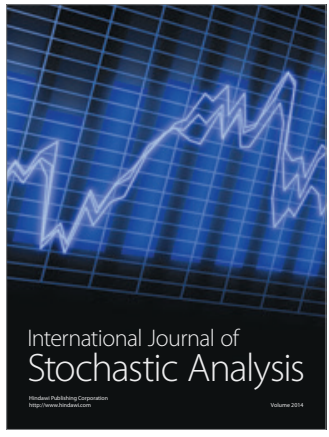

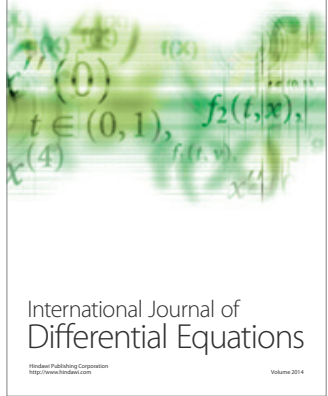
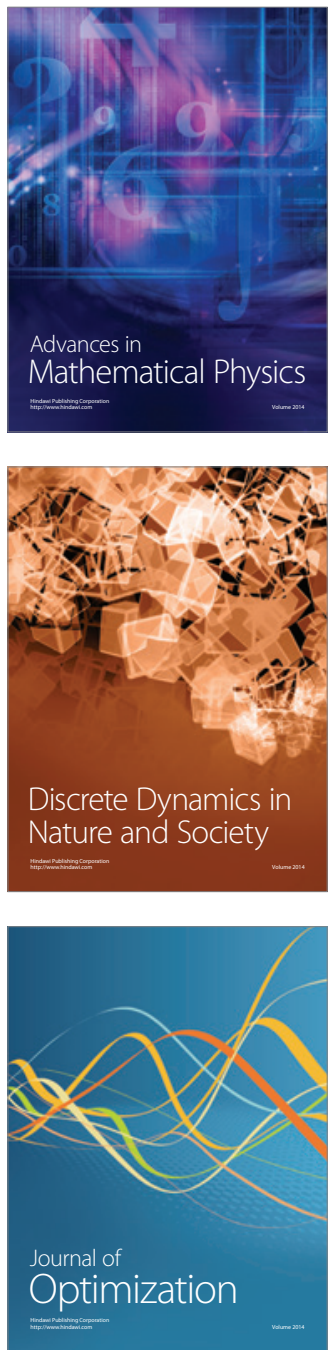